\title{
Image Registration for UAV Platform Based on KLT Features
}

\author{
J. LIU \& Z. C. HAO \& W. GAO \\ Changchun Institute of Optics, Fine Mechanics and Physics Chinese Academy of Sciences, Changchun, China
}

\begin{abstract}
Image registration technology is extremely significant in the virtual scene, automatic navigation, computer science, remote sensing image processing and many other aspects. It takes lots of difficulties for video tracking owing to the UAV platform is always unstable. In this paper, we utilized improved KLT algorithm to realize feature extraction, and used distance constraint to speed up matching, then applied the RANSAC to achieve precise matching, finally obtained the affine transform parameters. The experiment results demonstrate that this method can solve the problem of UAV camera shaking effectively.
\end{abstract}

KEYWORD: KLT; UAV; Image Registration; Feature point

\section{INSTRUCTION}

Almost one hundred years ago, the first Unmanned Aerial Vehicle (UAV) came out; the UAV has gone through three important periods which are airborne target drone, unmanned scout and all-purpose aircraft, especially since 90s, UAV has made brilliant contributions in the bight war, fox of desert, Kosovo war, and shown more and more significant efficiency and status in high tech wars.

The photo-electricity platform is the most basic assignment executing unit of the UAV, and the missions of UAV are accomplished depend on this platform mostly [1] [2] [3]. We can use photoelectricity platform and photo-electricity sensor to finish spying and surveillance battlefields, target capturing and recognizing, target survey and indication of weapon throwing in, campaign effect evaluation and so on. And that these are all based on the image and video processing capturing from the sensor.

Because of the influence of the air current and the shake of the platform itself when the UAV fly, the images are always blurred. This phenomenon makes the further processing (such as target tracking and target recognition) more difficult, therefore image Registration is important and necessary.

In this paper, we proposed a method which used KLT arithmetic to obtain feature points and used advanced RANSAC to wipe off the error matching to achieve the precise matching. Then we adopted Similarity model to get the transform parameters, after many experiments we concluded it can content the requests of motion compensation for the unstable video capturing platform.

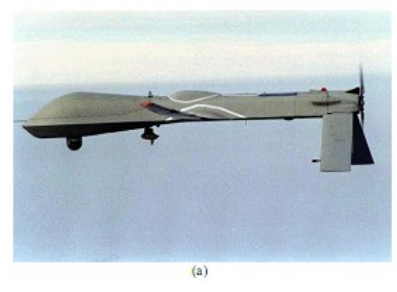

(a)Predator UAV

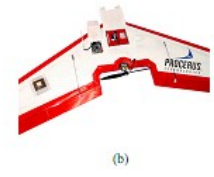

(b)Zagi UAV
Figure 1.UAVs loading photo-electricity platforms

\section{IMAGE REGISTRATION}

Simply speaking, the digital image registration is the best transform from one image to another [4]. The methods can divide into four types [5] [6] [7]: the one based on pixel correlation, feature, and model and transformation field.

Pixel information is the most intuitionistic representation notation for image, so there are many registration method based on it. The classis one is sequential similarity detection Algorithms [1] (SSDA) which was proposed by Barnea. This method has simple structure, fast speed, and is easy to realize. However, it's sensitive to noises and lowcontrast; meanwhile, it is not applicable to large angle rotation.

The primary way based on transform field is Fourier transform. The shift and rotation between the images waiting for matching can be fully reflected in 
frequency field. Because of the far executive speed of FFT, robustness of noise and low computation complexity, it is popular; its disadvantage is no solution to rotation due to its shift-invariability.

The method based on image features [8] [9] [10] [11] is the uppermost method for image registration, which can be applied to most situation. It has four steps as below:

1) Extract the feature set in two images;

2) According to some comparability rules, match between the two feature sets, and weed out outlines;

3) Estimate the transform model parameters using the matched feature pairs;

4) Map between images: map the target image to source image by the transform matrix.

For image registration based on feature-matching method, whether the features selected and matched correctly or not is the key point, so we used KLT [12] [13] (Kanada Lucas Tomasi) arithmetic which was the improvement of the one proposed by Lucas and Kanada, and in reference [4] it has been proved that it is inapplicable to move image pure gray detection, but for linear contorted affine transform it is pretty good.

\subsection{Feature extraction}

In practical application, image registration requests the features we extract should be invariable when different illumination and adapt to all circumstances. But no matter what method is used, the extracted features cannot conclude all moving information, such as horizontal gray edge can only represent the vertical component of change, therefore, many researchers committed themselves to solve this problem, many different features were proposed like corner, small image window which has higher frequency of special field, and the combination of some second-order partial derivatives. These are all based on the hypothesis of a 'good' feature, so maybe the features you extract are visual, but they are not always the good ones for matching and tracking.

In this paper, we use the KLT [12] [13] algorithm to feature extraction. The algorithm is based on the principled define of the feature quality: a 'good' feature is the one which can be tracked for the subsequent matching.

The rough procedure of KLT is that detecting eigenvalues of $2 * 2$ symmetrical gradient matrix to locate the feature windows.

The image in $\mathrm{t}+\mathrm{r}$ frame can be taken by moving every point in the $t$ frame image, like the equation (1).

$$
I(x, y, t+r)=I(x-\xi(x, y, t, r), y-\eta(x, x, t, r))
$$

The movement of point $\mathrm{X}=(\mathrm{x}, \mathrm{y})$ is $\delta=(\xi, \eta)$.
Then we can use equation (2) to descript that the point $\mathrm{X}$ in image $\mathrm{I}$ has moved to the point $\mathrm{AX}+\mathrm{d}$ in image $\mathrm{J}$.

$$
J(A X+d)=I(X)
$$

Here, $\mathrm{d}$ is the translation vector, and matrix $\mathrm{A}$ is for the transformation, such as affine or similar transform.

We need to make the cumulative variance $e$ (as shown in equation (3)) minimum.

$$
e=\iint_{W}[J(A X+d)-I(X)]^{2} w(X) d X
$$

$A$ and $d$ are what we want to solve for image registration, but the first step is to find some classify windows which have stable feature and can be detected.

We make taylor expansion to $\mathrm{J}(\mathrm{AX}+\mathrm{d})$, and ignore the high-order components, as (4)

$$
J(X+d)=J(X)+d \cdot g^{T}(X+d)
$$

Then take equation (4) to the equation (3) and make $A=1$, calculate the partial derivatives of $e_{\text {to }}$ $\mathrm{d}$, make it equal to 0 , finally we can get the equation (5) as below.

$$
Z d=e
$$

Namely,

$$
\begin{aligned}
& \iint_{W}\left[\begin{array}{cc}
g_{x}^{2} & g_{x} g_{y} \\
g_{x} g_{y} & g_{y}^{2}
\end{array}\right]\left[\begin{array}{l}
d_{x} \\
d_{y}
\end{array}\right] w(X) d X \\
& =\iint_{W}[I(X)-J(X)]\left[\begin{array}{l}
g_{x} \\
g_{y}
\end{array}\right] w(X) d X \\
& Z=\left[\begin{array}{cc}
g_{x}^{2} & g_{x} g_{y} \\
g_{x} g_{y} & g_{y}^{2}
\end{array}\right], g_{x}=\partial J / \partial x, g_{y}=\partial J / \partial y
\end{aligned}
$$

If the equation (6) has stable solution, we can track the window, so the $2 * 2$ symmetrical matrix $Z$ must be well-conditioned. To reduce the influence of noise, it is requested that the eigenvalues of matrix are all large. That one eigenvalue is small and another is big means the color change of the pixel is one-way, maybe horizontal edge; that the two all big means a corner, salt and pepper texture or other model which can be tracked. But in image processing, the color change is subject to the maximum of pixel, so the eigenvalue cannot be infinitely large, as equation (8) shown.

$$
\min \left(\lambda_{1}, \lambda_{2}\right)>\lambda
$$

$\lambda$ is the threshold of the eigenvalues.

Then the one whose eigenvalues satisfy the equation (8) is candidate feature point.

The feature extraction method we used in this paper can be realized by four steps: 
1) Image pre-processing to remove noise;

2) Divide the image to $M^{*} N$ blocks, and calculate the histogram of each block, set the maximum feature point number according to the gray complexity of each block;

3) To every point, calculate matrix Z, and estimate (8),and select the candidate points;

\subsection{The moving model we used}

We use affine transform model,

$$
\delta=D X+d
$$

$D=\left[\begin{array}{ll}d_{x x} & d_{x y} \\ d_{y x} & d_{y y}\end{array}\right]$ is transform matrix, and $A=1+D$

\subsection{Feature matching}

We calculate the partial derivatives of $e$ in equation (3) to $D$ and $d$, make it equal to 0 , that is, minimize the partial derivatives. Then the result as below:

$$
T z=a
$$

$$
\text { Here, } z^{T}=\left[d_{x x} d_{y x} d_{x y} d_{y y} d_{x} d_{y}\right],
$$$$
a=\iint_{W}[I(X)-J(X)]\left[\begin{array}{lllll}
x g_{x} & x g_{y} & y g_{x} & y g_{y} g_{x} & g_{y}
\end{array}\right]^{\mathrm{T}} w \cdot d x,
$$$$
T=\iint_{W}\left[\begin{array}{cc}
U & V \\
V^{T} & Z
\end{array}\right] w d x,
$$$$
\text { And, } U=\left[\begin{array}{cccc}
x^{2} g_{x}^{2} & x^{2} g_{x} g_{y} & x y g_{x}^{2} & x y g_{x} g_{y} \\
x^{2} g_{x} g_{y} & x^{2} g_{y}^{2} & x y g_{x} g_{y} & x y g_{y}^{2} \\
x y g_{x}^{2} & x y g_{x} g_{y} & y^{2} g_{x}^{2} & y^{2} g_{x} g_{y} \\
x y g_{x} g_{y} & x y g_{y}^{2} & y^{2} g_{x} g_{y} & y^{2} g_{y}^{2}
\end{array}\right] \text {, }
$$

$$
V^{T}=\left[\begin{array}{cccc}
x g_{x}^{2} & x g_{x} g_{y} & y g_{x}^{2} & y g_{x} g_{y} \\
x g_{x} g_{y} & x g_{y}^{2} & y g_{x} g_{y} & y g_{y}^{2}
\end{array}\right], Z=\left[\begin{array}{cc}
g_{x}^{2} & g_{x} g_{y} \\
g_{x} g_{y} & g_{y}^{2}
\end{array}\right]
$$

To get the $z^{T}=\left[d_{x x} d_{y x} d_{x y} d_{y y} d_{x} d_{y}\right]$, we use Newton- Raphson method repeatedly.

In order to accelerate the match speed, we use distance constraint, which means that the distance between matched point in image $\mathrm{J}$ and the point in image I must not be too big because the shift between points results from the UAV shake itself, and it's not too seriously. So using distance constraint can cut down lots of unnecessary matching communication.

And we use RANSAC [14] to reject the wrong matching and outlier points, the results are all right.

\section{EXPERIMENT RESULT}

Firstly, the experiments have been carried out by using standard pictures, as figure 2 shown, we can see no matter the background is simple or complex, the method is ok. And we also find out that the points which are the 'good' ones for tracking maybe some points which are not concerned by us, like the left-top of the figure2 (b).

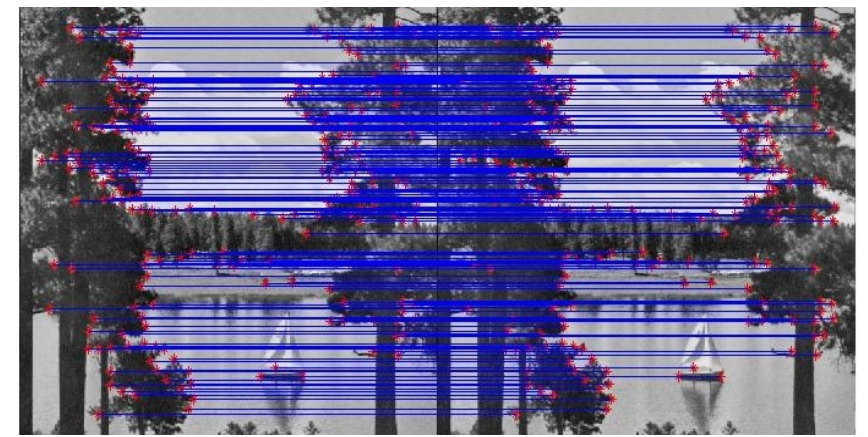

(a)Lake

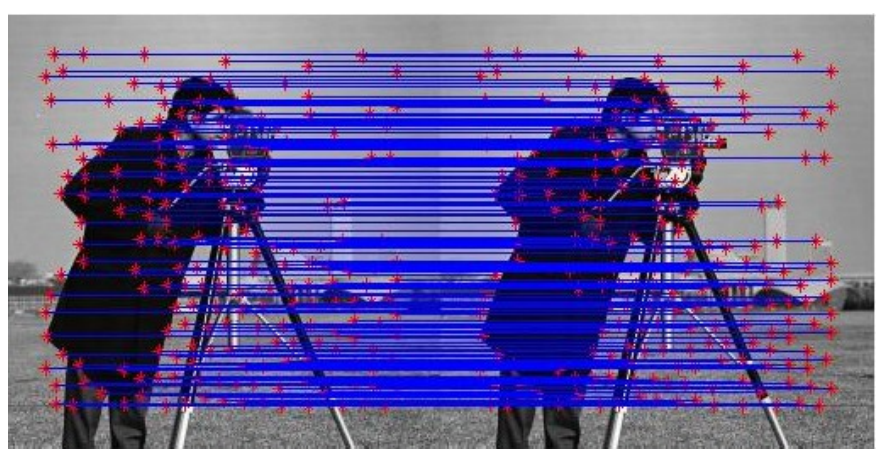

(b)Camera man

Figure 2. results using standard pictures

The figure 3 is the result of only using KLT without distance constraints and RANSAC, the video we use is the video [15] Zagi UAV tracking the ground vehicles. From figure 3, we can obviously find that there are some false matching (such as the left-bottom of the picture), and use the method we proposed in this paper, these false can be removed easily.

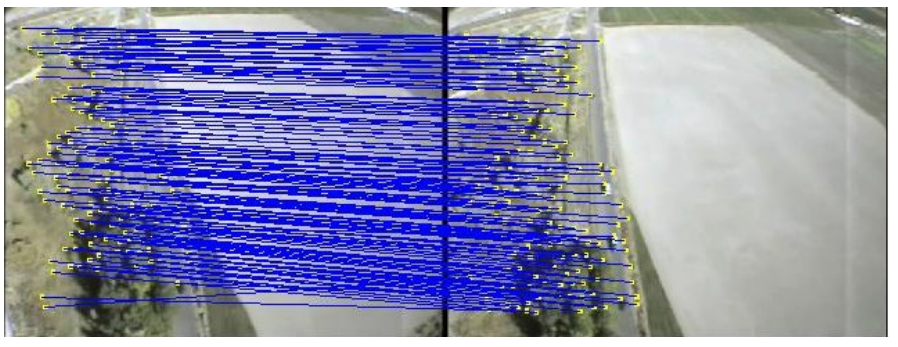

Figure 3. Matching result without any contraints

The figure $4(\mathrm{~b})$ is the difference between the current frame and registration image, it can be seen that the registration effect is very well. 


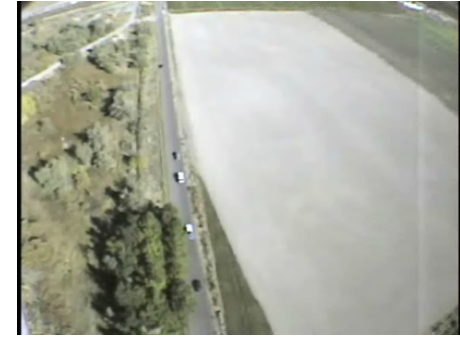

(a)registration image

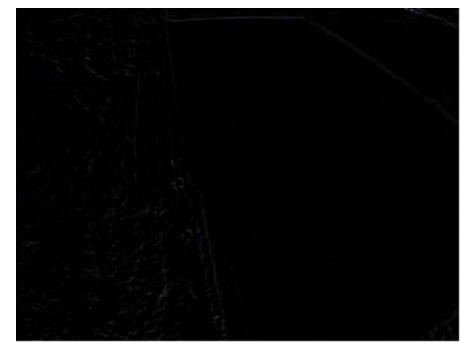

(b)the difference between registration and current image

Figure 4 UAVs loading photoelectricity platforms

\section{CONCLUTION}

In this paper, we utilized the improved KLT algorithm to realize feature extraction, then used distance constraint to speed up matching, simultaneously, applied the RANSAC to achieve precise matching, finally obtained the affine transform parameters. The experiment results demonstrate that this method can solve the problem of UAV camera shaking effectively.

\section{REFERENCES}

[1] CHENG G, Yang S L. 2005, A Summary of Airborne Photoelectronic Systems on Drones, Applied Optics, 26(4): 1-4.

[2] WANG M, TU D W, ZHOU X C. 2011 Moving object detection by combining SIFT and differential multiplication. Optics and Precision Engineering. 4:892899.

[3] JI H, WU Y H, SUN H H. 2009 SIFT feature matching algorithm with global information. Optics and Precision Engineering. 17:439-444.

[4] LI Q, JI H B, TONG M. 2011 Robust registration of multimodality medical images based on principalordinal feature and hybrid entropy. JOURNAL OF XIDIAN UNIVERSITY, 38 (6):34-42.

[5] SUN C. Algorithm Research on Image Registration and its applacation on document image registration. University of Electronic science and technology of china.2009.

[6] NIE Ho B, HOU Q Y, ZHAO M, ZHANG W. 2011 IR/visible image registration based on EM iteration of loglikelihood function. Optics and Precision Engineering. 19: 657-663.

[7] Yutaro Y, Hyoungseop K, and Akiyoshi Y. 2006 A Method for Image Registration by Maximization of Mutual Information. SICE-ICASE International Joint Conference, Bexco, Busan, Korea Oct. 18-21.

[8] YUAN X G, PENG G H, WANG L. 2011 GPU-based Real Time Image Registration with Variant SIFT. Computer Science, 38: 300-303.

[9] MA C J, GUAN P, LI R W. 2011 Image automatic mosaic method based on phase and SIFT feather correlation. LASER \& INFRARED, 41:470-473.

[10] Jonathan C, Anant M, Mark R, John T. 2007 A combined feature ensimble based mutual information scheme for robust inter-modal, inter-protocal image registration. 2007 4th IEEE International Symposium on Biomedical Imaging: From Nano to Macro, 12-15 April.

[11] Lin H, Du P J. 2010 Image Registration Based on Corner Detection and Affine Transformation. 3rd International Congress on Image and Signal Processing (CISP 2010) :2184-2188

[12] S. H. F. Barnea D.I, 1976 A class of algorithms for digital image registration. IEEE Transactions on Computers, 2: 179-186.

[13] Jianbo Shi and Carlo Tomasi 1994 Good Features to Track, IEEE Conference on Computer Vision and Pattern Recognition, 593-600, 1994.

[14] HUANG Y Q,FU Y,MA G K. Cylindrical panoramic image stitching method based on RANSAC algorithm. JOURNAL OF SHENYANG UNIVERSITY OF TECHNOLOGY. vol.30, 2008.

[15] Procerus TM Technologies, "UAV Convoy Following," http:// procerusuav. com/ gallery. Php 